\title{
Wave-Packet Scattering Without Kinematic Entanglement: Convergence of Expectation Values
}

\author{
Lawrence S. Schulman and Leonard J. Schulman
}

\begin{abstract}
The wave packet spread of a particle in a collection of different mass particles, all with Gaussian wave functions, evolves to a value that is inversely proportional to the mass of the particle. The assumptions underlying this result and its derivation are reviewed. A mathematical demonstration of the convergence of an iteration central to this assertion is presented. Finally, the question of in-principle measurement of wave packet spread is taken up.
\end{abstract}

Index Terms-Group theory, quantum theory, scattering.

\section{INTRODUCTION}

$\mathbf{I}$ $\mathrm{N}$ THIS paper, we present new proofs and review physical results related to potential high-precision atomic experiments probing the foundations of quantum mechanics. The new material in this paper is a presentation (at the level of gedanken experiment) of justification of the experimental feasibility of measurement of wave function spread, as well as a demonstration that a certain iteration, essential to the physical result, does indeed converge in expectation. The main physical arguments have been presented in a recently published paper and will be reviewed below.

In contrast to the many beautiful experiments of the last decades, where the standard quantum result was known and the challenge was its confirmation (with a lottery ticket for disconfirmation), for the experiments suggested by this work, the answer is not known. As will be explained below, there is reason to believe [1] that a gas of particles, given enough time to equilibrate (in a certain sense), will be best described by Gaussian wave functions having a specific relation between the values of the spread of each species of particle, where "spread" refers to position uncertainty (second moment of position).

Two assumptions lie behind these assertions, and, perhaps more than the calculations in [1], it is of interest to test those assumptions experimentally. First, theoretical work has suggested that in a decohering environment particle wave functions tend to become Gaussian [2]-[4], implying that this form is not just convenient for calculations but is what occurs in nature. Second,

Manuscript received April 1, 2004; revised May 26, 2004. This work was supported by the National Science Foundation under Grant PHY 0099471 and Grant CCR 0049092, by the National Science Foundation Institute for Quantum Information, and by the Okinawa Foundation under a grant.

L. S. Schulman is with the Physics Department, Clarkson University, Potsdam, NY 13699-5820 USA (e-mail: schulman@clarkson.edu).

L. J. Schulman is with the California Institute of Technology, Pasadena, CA 91125 USA (e-mail: schulman@caltech.edu).

Digital Object Identifier 10.1109/TNANO.2004.840141 there is the infamous issue of whether wave packets spread indefinitely. This question is addressed in [5], as well as in [3]. What is established in these references is that the density matrix of a particle in an active (decohering) environment tends to become diagonal in position space. We do not believe this completely resolves the wave-packet spreading issue, but we will proceed in this paper on the assumption that either it has been resolved or that it can be resolved. We remark that the history of this question goes back to the founding of quantum mechanics. In [5], portions of the Born-Einstein correspondence are quoted, with Einstein expressing his dissatisfaction with indefinite wave-packet spreading in the following terms: "One would then have to be very surprised if a star or a fly, seen for the first time, somehow appeared quasilocalized." 1

With the foregoing assumptions, [1] shows (as we review below) that, if in a gas of particles of masses $m_{i}, i=1,2, \ldots$ there is nontrivial scattering, the Gaussian wave functions of the particles will tend to have isotropic spreads, with $\sigma_{i}^{2} m_{i}$ independent of $i$ (where $\sigma$ is the position-space spread, " $\Delta x$ "). A further feature is that these values are such that there is no kinematic entanglement upon scattering.

In Section II, we briefly review [1]. Section III establishes the limiting expectation values of the spreads, justifying the conclusions (reached numerically) in [1]. The issue of whether wave function spread can be measured at all is the subject of Section IV. We end with a discussion.

\section{SCATTERING}

A pair of particles is described by the Gaussian wave function

$$
\begin{aligned}
\psi\left(p_{1}, p_{2}\right)= & \exp \left[-\left(p_{1}-k_{1}\right)^{\top} \sigma_{1}^{2}\left(p_{1}-k_{1}\right)+i p_{1}^{\top} a_{1}\right] \cdots \\
& \times \exp \left[-\left(p_{2}-k_{2}\right)^{\top} \sigma_{2}^{2}\left(p_{2}-k_{2}\right)+i p_{2}^{\top} a_{2}\right]
\end{aligned}
$$

where the indices refer to the particles, $k$ and $a$ are the central momenta and positions, $p, k$, and $a$ are column 3-vectors, $\sigma$ is a $3 \times 3$ symmetric positive definite matrix, normalization is ignored, $T$ indicates transpose, and $\hbar=1$. We focus on terms quadratic in $p_{1}$ and $p_{2}$ and define for this wave function

$$
Q_{I}\left(p_{1}, p_{2}\right) \equiv p_{1}^{\top} \omega_{1} p_{1}+p_{2}^{\top} \omega_{2} p_{2}
$$

the "initial" quadratic form, where for convenience we define $\omega \equiv \sigma^{2}$. The particles scatter (requiring appropriate $k$ and $a$ ), and we examine the final wave function. Introduce center of

${ }^{1}$ Quoted and translated in the article by E. Joos, in [5] 
mass and relative coordinates by $P_{\mathrm{CM}} \equiv p_{1}+p_{2}, p \equiv r_{2} p_{1}-$ $r_{1} p_{2}$ (conjugate to $\left.x \equiv x_{1}-x_{2}\right)$, where $r_{i} \equiv m_{i} /\left(m_{1}+\right.$ $\left.m_{2}\right)$. For the relative coordinate, a well-known result for offresonance scattering is that the wave function retains its original shape, to wit

$$
\psi(x, t)=\psi(x-v t, 0) e^{i E t}+\frac{1}{|x|} f_{k}(\hat{x}) \psi(x \hat{k}-v t, 0) e^{i E t}
$$

where $k$ is $\psi$ 's central momentum $E=k^{2} / 2 m$, the scattering occurs near $x=0$, and $f_{k}(\hat{x})$ is the scattering amplitude in the direction $\hat{x}$ ([6, Sec. 11.2]. After each scattering and for each participant, we trace out the coordinates of the other particle, so in reality we are working at the level of the density matrix. (Nevertheless, for each collision, we can work with wave functions by considering the density matrix to be a weighted average of pure states.) As a result, the wave function (3) will result in the selection (from the corresponding density matrix) of another Gaussian wave function going in some particular direction. Going back to the two-particle problem, the directions that are selected for particle \#1 and for particle \#2 are correlated by total momentum conservation. In particular, $P_{\mathrm{CM}}$ is conserved, while the relative momentum of the particles is rotated: $p_{\text {final }}=$ $R p_{\text {initial }}$ with $R \in \mathrm{SO}(3)$. Thus, the form of the post-scattering wave function (or of any wave function selected from the post-scattering density matrix) will be $\psi_{\text {initial }}\left(p_{1}^{\prime}, p_{2}^{\prime}\right)$, with prime indicating final value. The passage from initial to final values is: $\left(p_{1}, p_{2}\right)=\left(r_{1} P_{\mathrm{CM}}+p, r_{2} P_{\mathrm{CM}}-p\right) \longrightarrow\left(r_{1} P_{\mathrm{CM}}+\right.$ $\left.R p, r_{2} P_{\mathrm{CM}}-R p\right)=\left(p_{1}^{\prime}, p_{2}^{\prime}\right)$. The logarithm of the wave function is a polynomial with many terms, but, since we are only studying the progress of the spread of the Gaussian, we need only keep track of the pure quadratic part. Calling the final form of this $Q_{F}$, we obtain

$$
\begin{aligned}
Q_{F}=p_{1}^{\top}\{ & \left(r_{1}+r_{2} R\right) \omega_{1}\left(r_{1}+r_{2} R^{-1}\right) \\
& \left.+r_{2}^{2}(I-R) \omega_{2}\left(I-R^{-1}\right)\right\} p_{1} \\
& +p_{2}^{\top}\left\{\left(r_{2}+r_{1} R\right) \omega_{2}\left(r_{2}+r_{1} R^{-1}\right)\right. \\
& \left.+r_{1}^{2}(I-R) \omega_{1}\left(I-R^{-1}\right)\right\} p_{2} \\
& +2 p_{1}^{\top}\left\{\left(r_{1}+r_{2} R\right) r_{1} \omega_{1}\left(I-R^{-1}\right)\right. \\
& \left.+(I-R) r_{2} \omega_{2}\left(r_{2}+r_{1} R^{-1}\right)\right\} p_{2}
\end{aligned}
$$

where $I$ is the identity matrix. This is our first opportunity to view a surprising feature of the scattering of Gaussians: if it should happen that $r_{1} \omega_{1}=r_{2} \omega_{2}=$ const $\cdot I$, then the cross term in $p_{1}$ and $p_{2}$, which is to say the entangled part, vanishes. One might have thought that this kind of entangling, which we call kinematic since it arises from momentum conservation alone, would be an inevitable consequence of particle interaction. For Gaussian wave packets, however, it is not. Of course, the multiple outcomes of the experiment, as described by $f_{k}(\hat{x})$ of (3), do entail entanglement, so one has not escaped this phenomenon except in particular limits.

To determine the new spread of, say, particle \#1, we should integrate out the coordinates of particle \#2. In principle, this can introduce new terms quadratic in $p_{1}$ into the corresponding piece of $Q_{F}$. However, this turns out not to be the case, as shown in [1]. The new spread for \#1 is thus the expression sandwiched by $p_{1}^{\top}\{\ldots\} p_{1}$; similarly for $p_{2}$. Introducing the notation $\xi_{i} \equiv$ $r_{i} \omega_{i}$, we obtain the spread-iteration rule

$$
\begin{gathered}
\xi_{1}^{\prime}=\left(r_{1}+r_{2} R\right) \xi_{1}\left(r_{1}+r_{2} R^{-1}\right) \\
+r_{1} r_{2}(1-R) \xi_{2}\left(1-R^{-1}\right) \\
\xi_{2}^{\prime}=\left(r_{2}+r_{1} R\right) \xi_{2}\left(r_{2}+r_{1} R^{-1}\right) \\
+r_{1} r_{2}(1-R) \xi_{1}\left(1-R^{-1}\right) .
\end{gathered}
$$

Note that (5) preserves the symmetry and positivity of the $\omega$ 's (and $\sigma$ 's).

In [1], numerical methods were used to establish that the iteration (5) converges to $\xi_{1}^{\infty}=\xi_{2}^{\infty}=I \operatorname{Tr}\left(r_{1} \omega_{1}^{(0)}+r_{2} \omega_{2}^{(0)}\right) / 6$. This implies that $r_{1} \omega_{1}=r_{2} \omega_{2}=$ multiple of the identity, so that once this limit is approached there is a cessation of kinematic entanglement.

For future use, we present an alternative form of (5). Define $P \equiv \xi_{1}+\xi_{2}, M \equiv \xi_{1}-\xi_{2}$, and $\delta \equiv r_{1}-r_{2}$. Then

$$
\begin{aligned}
P^{\prime}= & \frac{1}{2}\left(P+R P R^{\top}\right)+\frac{\delta}{2}\left(M-R M R^{\top}\right) \\
M^{\prime}= & \frac{1}{2}\left(1-\delta^{2}\right)\left(R M+M R^{\top}\right) \cdots \\
& +\frac{1}{2} \delta^{2}\left(M+R M R^{\top}\right)+\frac{\delta}{2}\left(P-P R P^{\top}\right) .
\end{aligned}
$$

Remarks: 1) Note that $\operatorname{Tr}\left(\xi_{1}+\xi_{2}\right)$ is preserved under the iteration. 2) Physically, we should look at a slight variant of (5): take there to be collections of particles of type-1 and type-2, each with a distribution of spreads. Then it is the convergence of these distributions that would correspond to what goes on in a gas. This form of the iteration has been checked numerically and gives the same result as the simpler version. 3) The convergence to a common limit obtains even if there are more than two types of particle. 4) Convergence is obtained for a variety of selection schemes (probability measures) for $R$. Exceptional situations can arise when $R$ is taken from an insufficiently comprehensive subset of $\mathrm{SO}(N)$.

\section{ITERATION}

Convergence of (5) is far more general than what is required for the physical result. It holds for all dimensions $N$ (with $R \in$ $\mathrm{SO}(N)$ ) and for antisymmetric as well as symmetric $\xi$ 's (there are peculiarities in one and two dimensions which we will describe). It even works if separate rotations are applied to $\xi_{1}$ and $\xi_{2}$, although we will not consider that case.

We now show that, if $R$ is selected with Haar measure, the expectation values of the $\xi$ 's converge.

\section{A. Dimension Three or Greater}

Let $\xi_{k}, k=1,2$, be arbitrary $N \times N$ real matrices and $R \in$ $\mathrm{SO}(N)$ with $N \geq 3$. Then, under the iteration (5) (with the implicit 3 replaced by $N$ ), the expectations of both $\xi_{k}$ 's converge to $\operatorname{Tr}\left(\xi_{1}+\xi_{2}\right) I / 2 N$, with $I$ the $N \times N$ identity.

Let $\lambda(\alpha, \beta, R) \equiv \alpha I+\beta R^{\top}$. Define a linear operator $\Lambda$ on $N \times N$ matrices, by $\Lambda(\alpha, \beta, R) \xi \equiv \lambda^{\top} \xi \lambda$. Further let $A_{1}(R) \equiv \Lambda\left(r_{1}, r_{2}, R\right), A_{2}(R) \equiv \Lambda\left(r_{2}, r_{1}, R\right)$ and 
$B(R) \equiv \Lambda\left(\sqrt{r_{1} r_{2}},-\sqrt{r_{1} r_{2}}, R\right)$, with, as usual, $r_{1}+r_{2}=1$. The $n$th iterate of (5) can be written

$$
\left(\begin{array}{l}
\xi_{1}^{(n)} \\
\xi_{2}^{(n)}
\end{array}\right)=\prod_{\ell=1}^{n}\left(\begin{array}{cc}
A_{1}\left(R_{k}\right) & B\left(R_{k}\right) \\
B\left(R_{k}\right) & A_{2}\left(R_{k}\right)
\end{array}\right)\left(\begin{array}{c}
\xi_{1}^{(0)} \\
\xi_{2}^{(0)}
\end{array}\right)
$$

where an ordered product is understood. Since (5) preserves the trace of the sum of the $\xi_{k}$, all that must be shown is that the $\xi_{k}$ converge to a multiple of the identity. We focus on $\xi_{1}$.

By expanding the product, one can see that both $\xi_{1}^{(0)}$ and $\xi_{2}^{(0)}$ are acted upon by products of $A$ 's and $B$ 's $-n$ of them in each product. Each $\xi_{\ell}^{(0)}(\ell=1,2)$ is acted on by $2^{n-1}$ of these products, which are summed to give the final result. Thus

$$
\begin{aligned}
\xi_{1}^{(n)}=[ & A_{1}\left(R_{n}\right) A_{1}\left(R_{n-1}\right) \ldots A_{1}\left(R_{1}\right) \\
& \left.+A_{1}\left(R_{n}\right) B\left(R_{n-1}\right) \ldots A_{1}\left(R_{1}\right)+\ldots\right] \xi_{1}^{(0)} \\
& +\left[B\left(R_{n}\right) A_{1}\left(R_{n-1}\right) \ldots A_{2}\left(R_{1}\right)\right. \\
& \left.+B\left(R_{n}\right) B\left(R_{n-1}\right) \ldots A_{2}\left(R_{1}\right)+\ldots\right] \xi_{2}^{(0)}
\end{aligned}
$$

Each term in (9) has the form

$$
\begin{array}{r}
\eta^{(n)}=\Lambda\left(\alpha_{n}, \beta_{n}, R_{n}\right) \cdot \Lambda\left(\alpha_{n-1}, \beta_{n-1}, R_{n-1}\right) \cdots \\
\cdot \Lambda\left(\alpha_{1}, \beta_{1}, R_{1}\right) \xi^{(0)}
\end{array}
$$

with $\xi^{(0)}$ either $\xi_{1}^{(0)}$ or $\xi_{2}^{(0)}$. From (9), it follows that the leftmost factor (indexed by $n$ ) must be either $A_{1}$ or $B$ and the rightmost (indexed by 1) must be $A_{1}$ or $B$ for $\xi_{1}^{(0)}$ and $A_{2}$ or $B$ for $\xi_{2}^{(0)}$. Because the $R$ 's are independent random variables, we can unravel the expectation term by term. Consider

$$
\begin{aligned}
\left\langle\eta^{(1)}\right\rangle= & \left\langle\Lambda\left(\alpha_{1}, \beta_{1}, R_{1}\right) \xi^{(0)}\right\rangle \\
= & \alpha_{1}^{2} \xi^{(0)}+\beta_{1}^{2}\left\langle R_{1} \xi^{(0)} R_{1}^{\top}\right\rangle \\
& +\alpha_{1} \beta_{1}\left\{\left\langle R_{1} \xi^{(0)}\right\rangle+\left\langle\xi^{(0)} R_{1}^{\top}\right\rangle\right\}
\end{aligned}
$$

where the brackets, " $\langle\cdot\rangle$ " indicate expectation with respect to Haar measure on $\mathrm{SO}(N)$. To evaluate (11), we establish the following:

$$
\langle R\rangle=\kappa(N) I, \quad \text { with } \kappa(N) \equiv \begin{cases}0, & N \text { even } \\ 1 / N, & N \text { odd }\end{cases}
$$

and, for $N>2$,

$$
\left\langle R \xi R^{\top}\right\rangle=I \operatorname{Tr}(\xi) / N
$$

where $\xi$ is an arbitrary $N \times N$ matrix.

Proof of (12): For $N$ even, both $R$ and $-R$ are in $\mathrm{SO}(N)$, so that $\langle R\rangle=0$ follows by symmetry. For odd $N, R$ generically has one eigenvalue 1 , with the others coming in complex conjugate pairs. $R$ can therefore be written $R=P_{R}+Q$ with $P_{R}$ a projection on a one-dimensional (1-D) subspace and $Q$ acting as an orthogonal transform on the complimentary $(N-1)$-dimensional space (hence $Q \in \mathrm{SO}(N-1)$ ). We perform the $\mathrm{SO}(N-1)$ average by holding the $P_{R}$ fixed and averaging over $Q$. The latter (an $\mathrm{SO}(N-1)$ average) gives zero, so that the average over all $R \in \mathrm{SO}(N)$ becomes an average over projections $P_{R}$. This average has no preferred direction and must therefore be a multiple of the identity. To find the multiplier, use an arbitrary normalized vector $p$ and evaluate $\left\langle p^{\top} P_{R} p\right\rangle$. The result must be the same whether we average over directions of $P_{R}$ or hold $P_{R}$ fixed and average over $p$. However, then the value will be the expectation of the square of a particular component of a random $p$. This will be $1 / N$ of the total normalization, namely $1 / N$.

Proof of (13): For each $\xi, \zeta \equiv\left\langle R \xi R^{\top}\right\rangle$ is an $N \times N$ matrix. For any $R_{0} \in \mathrm{SO}(N), \zeta=R_{0} \zeta R_{0}^{\top}$, by virtue of the translational invariance of the measure. This also implies that $\zeta$ commutes with all generators of $\mathrm{SO}(N)$, and that if $u$ is an eigenvector of $\zeta$, then so is $R u$, with the same eigenvalue, for any $R \in \mathrm{SO}(N)$. Since conjugation preserves symmetry or antisymmetry of a matrix, we examine the two cases, $\xi$ symmetric or antisymmetric separately.

Suppose $\xi$ and (hence) $\zeta$ are antisymmetric. Then $\zeta$ can be expressed as a sum of generators of $\mathrm{SO}(N)$. However, the only situation where it can commute with all of them is $N=2$, for which there is but a single generator. (The $N=2$ case will be treated below.) Thus, for $N>2, \zeta$ must be zero.

Now suppose $\xi$ and (hence) $\zeta$ are symmetric. Then the real symmetric $\zeta$ can be fully diagonalized with real eigenvalues and eigenvectors. Write it as $\zeta=\sum z_{\ell} u_{\ell} u_{\ell}^{\dagger}$. The $u$ 's can be taken to be an orthonormal set and can be rotated into one another by appropriate elements of $\mathrm{SO}(N)$. Hence, all must have the same eigenvalue. However, this implies that $\zeta$ is proportional to $I$, the identity. Since conjugation preserves the trace, we obtain the value given in (13).

Returning to (11), we obtain

$$
\begin{aligned}
\left\langle\eta^{(1)}\right\rangle & =\left[\alpha_{1}^{2}+2 \alpha_{1} \beta_{1} \kappa(N)\right] \xi^{(0)}+\beta_{1}^{2} I \operatorname{Tr} \xi^{(0)} / N \\
& =\gamma_{1}^{2} \xi^{(0)}+\beta_{1}^{2} \iota x
\end{aligned}
$$

where we use the notation $\iota \equiv I / N, \gamma_{\ell}^{2}=\alpha_{\ell}^{2}+2 \alpha_{\ell} \beta_{\ell} \kappa(N)$, and $x \equiv \operatorname{Tr} \xi^{(0)}$. Note that for $0<r_{1}<1$, for all $\alpha$, etc. (in $A_{1}, A_{2}$, and $B), \gamma$ is strictly less than one. Next act on this expression with $\Lambda\left(\alpha_{2}, \beta_{2}, R_{2}\right)$ and again average over the group. A short calculation gives

$$
\begin{aligned}
\left\langle\eta^{(2)}\right\rangle & =\left\langle\left(\alpha_{2}+\beta_{2} R_{2}\right)\left[\gamma_{1}^{2} \xi^{(0)}+\beta_{1}^{2} \iota x\right]\left(\alpha_{2}+\beta_{2} R_{2}^{\top}\right)\right\rangle \\
& =\gamma_{1}^{2}\left(\gamma_{2}^{2} \xi^{(0)}+\beta_{2}^{2} \iota x\right)+\beta_{1}^{2} \iota x\left(\gamma_{2}^{2}+\beta_{2}^{2}\right)
\end{aligned}
$$

by obvious application of (12) and (13). Brackets in (15) indicate average with respect to $R_{2}$, or both $R_{1}$ and $R_{2}$, as appropriate. This can be rewritten as

$$
\left\langle\eta^{(2)}\right\rangle=\gamma_{1}^{2} \gamma_{2}^{2}\left(\xi^{(0)}-\iota x\right)+\iota x\left(\gamma_{1}^{2}+\beta_{1}^{2}\right)\left(\gamma_{2}^{2}+\beta_{2}^{2}\right) .
$$

Continuing, the term involving $\xi^{(0)}-\iota x$ acquires a longer and longer string of $\gamma$ 's, all less than unity. Moreover, since $\beta^{2}$ is strictly positive (provided neither $r_{1}$ nor $r_{2}$ is zero), the ratio $\gamma^{2} /\left(\gamma^{2}+\beta^{2}\right)$ (for any subscript) is bounded away from unity. It follows that, for each $\eta$, the first term, which has the long string of $\gamma$ 's, vanishes relative to the second term. The remaining expression is $\iota x$ times the product of the sum of the squares of the $\alpha$ 's and $\beta$ 's associated with the specific term. 
Since the surviving term is a multiple of the identity, we have established most of our desired result. The actual value of the sum of the multipliers of $\xi_{1}$ and $\xi_{2}$ (the limiting values) is fixed by the constancy $\operatorname{Tr}\left(\xi_{1}+\xi_{2}\right)$ under the iteration rule. We address their relative sizes in a moment, but first show how the $2^{n}$ terms contributing to $\left\langle\xi_{1}^{(n)}\right\rangle$ manage to give an order unity number. For simplicity, assume that the initial $\xi$ 's have the same trace so that all terms in (9) can be treated without constraints.

Gathering all summands in (9), terms of type $A$ contribute $r_{1}^{2}+2 r_{1} r_{2} \kappa+r_{2}^{2}=1-2 r_{1} r_{2}(1-\kappa)$, while type- $B$ terms yield $2 r_{1} r_{2}(1-\kappa)$. Suppose there are $k$ type- $A$ terms and $n-k$ type- $B$; then the sum is

$$
\sum\left(\begin{array}{l}
n \\
k
\end{array}\right)\left[1-2 r_{1} r_{2}(1-\kappa)\right]^{k}\left[2 r_{1} r_{2}(1-\kappa)\right]^{n-k}=1
$$

To study the multipliers of $I$, we return to (6) and (7). Since both $P$ and $M$ tend to multiples of the identity, the expectation of (7) (with $m$ defined by $M=m I$ ) simplifies to

$$
m^{\prime}=\left[\delta^{2}+\left(1-\delta^{2}\right) \kappa(N)\right] m .
$$

Noting that $\delta^{2}+\left(1-\delta^{2}\right) \kappa(N)=1-4 r_{1} r_{2}(1-\kappa)$, it follows that $m$ converges to zero, and both $\xi$ 's have the same limiting value, namely $I \operatorname{Tr}\left(\xi_{1}+\xi_{2}\right) / 2 N$.

\section{B. Dimension One}

The 1-D case allows complete analytic solution. The iteration is obtained by letting " $R$ " $=-1$ in (5) [7], but we generalize this slightly, taking $R=\exp (i \theta)$ as follows:

$$
\begin{aligned}
\xi_{1}^{\prime}= & \left(r_{1}+r_{2} e^{i \theta}\right) \xi_{1}\left(r_{1}+r_{2} e^{-i \theta}\right) \\
& +r_{1} r_{2}\left(1-e^{i \theta}\right) \xi_{2}\left(1-e^{-i \theta}\right) \\
\xi_{2}^{\prime}= & \left(r_{2}+r_{1} e^{i \theta}\right) \xi_{2}\left(r_{2}+r_{1} e^{-i \theta}\right) \\
& +r_{2} r_{1}\left(1-e^{i \theta}\right) \xi_{1}\left(1-e^{-i \theta}\right) .
\end{aligned}
$$

The joint evolution of $\left\{\xi_{1}, \xi_{2}\right\}$ (which are now numbers) is simple: adding the equations of (19), one finds $\xi_{1}+\xi_{2}=$ const, while subtraction shows that $\xi_{1}-\xi_{2}$ is multiplied on successive iterations by

$$
\begin{aligned}
\nu & \equiv 1-4 r_{1} r_{2}(1-\cos \theta) \\
& =\cos ^{2} \frac{\theta}{2}+\sin ^{2} \frac{\theta}{2} \cos 4 \phi \\
& =\delta^{2}+\left(1-\delta^{2}\right) \cos \theta
\end{aligned}
$$

where $r_{1}=\cos ^{2} \phi .|\nu|$ is manifestly one or less. $\nu=1$ only if $\theta=0$ (nothing happens, i.e., no scattering) or if $\cos 4 \phi=1$, implying that one of the masses is zero. The case $\nu=-1$ can occur only if $\theta=\pi$ and $\cos 4 \phi=-1$, which corresponds to equal masses. In the latter situation, $\xi_{1}$ and $\xi_{2}$ exchange values on successive scatterings.

\section{Dimension Two}

The steps leading to (11) go through without change. Write $R \in \mathrm{SO}(2)$ as $R=\exp (\psi J)$, with

$$
J=\left(\begin{array}{cc}
0 & -1 \\
1 & 0
\end{array}\right) .
$$

The expectation is the integral $(1 / 2 \pi) \int_{0}^{2 \pi} d \psi$, so that $\langle R\rangle=0$. The matrix $\xi$ (dropping the superscript 0 ) is conveniently represented as

$$
\xi=I+y J+\left(\begin{array}{cc}
z & x \\
x & -z
\end{array}\right), \quad x, y, z \in \mathbb{R} .
$$

To evaluate $\left\langle R \xi R^{\top}\right\rangle$, we note that $R$ commutes with $J$, leading to

$$
\begin{aligned}
R \xi R^{\top}= & I+y J \\
& +\left(\begin{array}{cc}
z \cos 2 \psi-x \sin 2 \psi & x \cos 2 \psi+z \sin 2 \psi \\
x \cos 2 \psi+z \sin 2 \psi & -z \cos 2 \psi+x \sin 2 \psi
\end{array}\right) .
\end{aligned}
$$

Clearly integration over $\psi$ will eliminate all but $I+y J$. As a result, the two-dimensional (2-D) version of (14) is

$$
\begin{aligned}
\left\langle\eta^{(1)}\right\rangle & =\alpha_{1}^{2} \xi^{(0)}+\beta_{1}^{2}(I+y J) \\
& =\left(\alpha_{1}^{2}+\beta_{1}^{2}\right)(I+y J)+\alpha_{1}^{2}\left(\begin{array}{cc}
z & x \\
x & -z
\end{array}\right) .
\end{aligned}
$$

As before, the fact that $\beta^{2}$ is bounded away from zero implies that the multiplier of $x$ and $z$ will disappear as additional applications of the operators $\Lambda_{\ell}$ occur. Thus, unlike other dimensions, for $N=2$, the limits of both $\xi$ 's are not simply the identity, but preserve the original value of " $y$ " in the representation (21). However, the limiting objects do commute with rotations, and it becomes convenient to return to the sum and difference representation, (6) and (7). It is obvious that, if $P$ starts as, say, $P=p_{0} I+p_{1} J$, then those coefficients are unchanged after any number of iterations. For $M$, things are a bit more involved. Let $M=m_{0} I+m_{1} J ;$ a simple calculation leads to

$$
M^{\prime}=m_{0}^{\prime} I+m_{1}^{\prime} J=\left(m_{0} I+m_{1} J\right)\left(\delta^{2}+\left(1-\delta^{2}\right) \cos \psi\right)
$$

where we have used the fact that $R+R^{\top}=2 I \cos \psi$. Except for the case $r_{1}=r_{2}=1 / 2$, the factor on the right is generically less than one, and $M \rightarrow 0$. It follows that if

$$
\xi_{\ell}^{(0)}=x_{\ell} I+y_{\ell} J+\left(\begin{array}{cc}
z_{\ell} & x_{\ell} \\
x_{\ell} & -z_{\ell}
\end{array}\right), \quad \ell=1,2
$$

then

$$
\xi_{\ell}^{(\infty)}=\frac{1}{2}\left[\left(x_{1}+x_{2}\right) I+\left(y_{1}+y_{2}\right) J\right], \quad \ell=1,2 .
$$

\section{An Exceptional Case (In Two Dimensions)}

The result (26) holds for all nonzero $r$ values. However, that expression addresses only expectations. Under an iteration, this expectation may not coincide with the iterate. Numerically we have found that this contingency does not arise except for the 
case $r=1 / 2$. For dimension-two and equal masses, part of the noncommuting piece of the initial conditions also survives. This is a sort of interpolation between dimensions 1 and 3: in dimension-1, the spreads flip back and forth (for $r=1 / 2$ ), while in dimension-3 they become the same.

In this case $(\delta=0),(6)$ and (7) take the form

$$
\begin{aligned}
P^{\prime} & =\frac{1}{2}\left(P+R P R^{\top}\right) \\
M^{\prime} & =\frac{1}{2}\left(R M+M R^{\top}\right) .
\end{aligned}
$$

Initial conditions are as in (25), implying that $P$ and $M$ have the same form

$$
\begin{gathered}
P=p_{0} I+p_{1} J+\left(\begin{array}{cc}
p_{3} & p_{2} \\
p_{2} & -p_{3}
\end{array}\right) \\
M=m_{0} I+m_{1} J+\left(\begin{array}{cc}
m_{3} & m_{2} \\
m_{2} & -m_{3}
\end{array}\right) .
\end{gathered}
$$

The next task is to evaluate $R P R^{\top}$ and integrate over the rotation angle. This is straightforward and the $p_{2}$ and $p_{3}$ terms drop out. The case $\left(R M+M R^{\top}\right)$ is more interesting. Part of this calculation is the same as the $r_{1} \neq 1 / 2$ case, but, for the $r=1 / 2$ case, the coefficients $m_{2}$ and $m_{3}$ do not drop out. It is easy to verify that, if the rotation is through an angle $\psi$, the new $m_{2}$ and $m_{3}$ values are given in terms of the old by

$$
\left(\begin{array}{l}
m_{2}^{\prime} \\
m_{3}^{\prime}
\end{array}\right)=\left(\begin{array}{cc}
\cos \psi & -\sin \psi \\
\sin \psi & \cos \psi
\end{array}\right)\left(\begin{array}{l}
m_{2} \\
m_{3}
\end{array}\right) .
$$

That is, they are rotated. As a result, for the asymptotic value of $M$ the $m_{0}$ and $m_{1}$ terms do conveniently disappear, but

$$
\left(\begin{array}{l}
m_{2}^{(n)} \\
m_{3}^{(n)}
\end{array}\right)=\left(\begin{array}{cc}
\cos \left(\sum \psi\right) & -\sin \left(\sum \psi\right) \\
\sin \left(\sum \psi\right) & \cos \left(\sum \psi\right)
\end{array}\right)\left(\begin{array}{l}
m_{2}^{(0)} \\
m_{3}^{(0)}
\end{array}\right) .
$$

Combining the pieces for this $r=1 / 2$ case, if the initial form of the $\xi$ 's is given by (25), then, after sufficiently large $n$, one gets

$$
\begin{aligned}
\xi_{\ell}^{(\infty)}=\frac{1}{2}\left[\left(x_{1}+\right.\right. & \left.\left.x_{2}\right) I+\left(y_{1}+y_{2}\right) J\right] \\
& -(-1)^{\ell}\left(\begin{array}{cc}
u^{(n)} & v^{(n)} \\
v^{(n)} & -u^{(n)}
\end{array}\right), \quad \ell=1,2
\end{aligned}
$$

where

$$
\begin{aligned}
& u^{(0)}=\frac{1}{2} \operatorname{Tr}\left[\left(\begin{array}{cc}
1 & 0 \\
0 & -1
\end{array}\right)\left(\xi_{1}^{(0)}-\xi_{2}^{(0)}\right)\right] \\
& v^{(0)}=\frac{1}{2} \operatorname{Tr}\left[\left(\begin{array}{ll}
0 & 1 \\
1 & 0
\end{array}\right)\left(\xi_{1}^{(0)}-\xi_{2}^{(0)}\right)\right]
\end{aligned}
$$

and $u^{(n)}$ and $v^{(n)}$ are the result of rotating the initial $u$ and $v$ values through the sum of the angles of all rotations.

\section{MEASUREMENT OF SPREAD}

How does one measure spread? If one measures " $x$ " for a large number of particles in a gas, it is difficult to separate wavefunction spread from the spread in central position. That is, in terms of the quantum operator $\hat{x}$, spread is $(\Delta x)^{2}=\left\langle(\hat{x}-\bar{x})^{2}\right\rangle$, where $\bar{x} \equiv\langle\hat{x}\rangle$. However, if all one measures is $\hat{x}$ and if the central position of the packet is not known a priori, then this definition will reflect a lack of information both about the central position of the packet as well as the spread of its wave function.

Our goal in the present discussion is not to offer an optimal way to measure $(\Delta x)^{2}$, only to give an in-principle argument to show that measurements can be sensitive to the spread of an individual wave function.

Imagine the particles in a container, one of whose walls is suddenly removed. A two-slit interference device is located far enough away for the interval of arrival times to bracket a momentum range and direction. If the spread transverse to the relatively well-defined direction of motion is less than the slit separation, there will be no diffraction pattern. If it is larger, there can be such a pattern. The fact that we have not measured the transverse position (whose nonquantum uncertainty ${ }^{2}$ may well exceed the slit separation) does not destroy the interference pattern because the maxima and minima of the pattern only depend on the distances from the slits to the screen. ${ }^{3}$ It is easy to see that the condition for this measurement to succeed (by not allowing the size of the container to destroy the interference pattern) is

$$
\frac{D}{L} \ll \frac{\lambda}{d}
$$

where $D$ is the transverse dimension of the container in which the particles are originally found, $L$ the distance to the slits from that container, $d$ the slit separation, and $\lambda$ the (de Broglie) wavelength of the particles. For an experiment involving two kinds of particle the presence or absence of a diffraction pattern must be separately established for each particle type. A variety of strategies can be employed to this end, for example, "screens" (i.e., position detectors) preferentially sensitive to one or the other particle.

\section{Discussion}

In this paper, we have mostly confined our discussion to the limit of the expectation values of the iteration using Haar measure on $\mathrm{SO}(N)$, although we did display one instance where expectation and limit did not agree. Numerically we have found that the two-dimension equal-mass case is the only instance; nevertheless, a proof of actual convergence (when it occurs) would be preferable and is planned.

An experimental study of the predicted mass-to-spread relation offers an opportunity to explore one of the muddier issues in the foundations of quantum mechanics. Our demonstration in Section II depended on nothing interesting happening with respect to spontaneous wave-packet spreading during the time of collision. Moreover, convergence to the mass-to-spread relation requires several scatterings (depending on the mass ratio), so that the stabilization of wave-packet spread is assumed for this longer time interval as well. An observation that spreads do

\footnotetext{
${ }^{2}$ This "uncertainty" is due to the variation in the center of the wave packet, and corresponds to the "central position spread' mentioned above

${ }^{3}$ Persistence of the interference pattern does not obtain when the transverse spread is too small; the amplitude from the second slit is just plain zero
} 
indeed satisfy the predicted relation would be as well a probe of these underlying assumptions.

There is an interesting entropy-information perspective on the extinguishing of kinematic entanglement. In the following, a particular particle after a collision, one traces out the coordinates of the other particle (as we did above), thereby, in general, increasing the von Neumann entropy. The equilibration of spread, as calculated here, is a kind of maximizing of entropy, in that the trace operation no longer generates an increase.

Finally, we exhibited an experimental arrangement where, in principle, spread in a packet, " $\Delta x$ " (as opposed to spread or uncertainty in the packet's center) can be deduced.

\section{ACKNOWLEDGMENT}

The authors would like to thank R. Balian and P. Pechukas for helpful discussions.

\section{REFERENCES}

[1] L. S. Schulman, "Evolution of wave-packet spread under sequential scattering of particles of unequal mass," Phys. Rev. Lett., vol. 92, p. 210404 , 2004.

[2] W. H. Zurek, S. Habib, and J. P. Paz, "Coherent states via decoherence," Phys. Rev. Lett., vol. 70, pp. 1187-1190, 1993.

[3] M. Tegmark, "Apparent wave function collapse caused by scattering," Found. Phys. Lett., vol. 6, pp. 571-590, 1993.

[4] M. Tegmark and H. S. Shapiro, "Decoherence produces coherence states: An explicit proof for harmonic chains," Phys. Rev. E, Stat. Phys. Plasmas Fluids Relat. Interdiscip. Top., vol. 50, pp. 2538-2547, 1994.

[5] D. Giulini, E. Joos, C. Kiefer, J. Kupsch, I.-O. Stamatescu, and H. D. Zeh, Decoherence and the Appearance of a Classical World in Quantum Theory. Berlin, Germany: Springer, 1996.
[6] E. Merzbacher, Quantum Mechanics, 2nd ed. New York: Wiley, 1970.

[7] L. S. Schulman, "Bounds on decoherence and error," Phys. Rev. A, Gen. Phys., vol. 57, pp. 840-844, 1998.

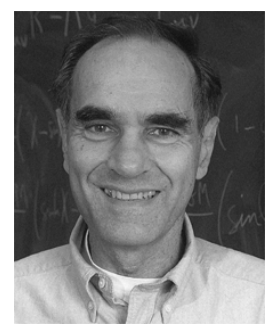

Lawrence S. Schulman did his undergraduate work at Yeshiva University and his graduate work at Princeton University, Princeton, NJ.

From 1967 to 1978 , he was on the faculty of Indiana University and, from 1970 to 1988 , with the Technion-Israel Institute of Technology, Haifa. Since 1985, he has been with Clarkson University, Potsdam, NY.

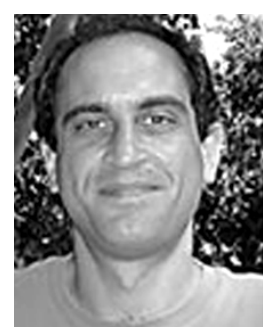

Leonard J. Schulman received the B.Sc. degree in mathematics and the Ph.D. degree in applied mathematics both from the Massachusetts Institute of Technology (MIT), Cambridge, in 1988 and 1992, respectively.

He has held appointments at the University of California, Berkeley, the Weizmann Institute of Science, the Georgia Institute of Technology, Atlanta, and the Mathematical Sciences Research Institute. Since 2000, he has been on the faculty of the California Institute of Technology, Pasadena.

Dr. Schulman was the recipient of the Jon A. Bucsela Prize in Mathematics from MIT, a National Science Foundation (NSF) Mathematical Sciences Postdoctoral Fellowship, an NSF CAREER Award, and an Okawa Foundation Grant. 